\title{
Hydrogenic Rydberg Atoms in Strong Magnetic Fields: Theoretical and Experimental Spectra in the Transition Region from Regularity to Irregularity *
}

\author{
A. Holle, G. Wiebusch, J. Main, and K.H. Welge \\ Fakultät für Physik, Universität Bielefeld, Bielefeld, Federal Republic of Germany
}

G. Zeller, G. Wunner, T. Ertl, and H. Ruder

Lehrstuhl für Theoretische Astrophysik, Universität Tübingen, Tübingen, Federal Republic of Germany

Received December 5, 1986

\begin{abstract}
For deuterium Rydberg atoms in a magnetic field of $\sim 6 \mathrm{~T}$ we compare the complete experimental spectrum in the range $-190 \mathrm{~cm}^{-1}$ to $-20 \mathrm{~cm}^{-1}$ with the positions and oscillator strengths of the corresponding quantum theoretically calculated photoabsorption lines. The agreement is excellent. The range of energy covered extends from the end of the $\ell$-mixing regime up to the regions where the approximate integrability of the problem is completely lost, and the corresponding classical system undergoes a transition to chaos.
\end{abstract}

PACS: $31.20 . \mathrm{D} ; 31.50 ; 32.60 . \mathrm{V}$

\section{Introduction}

The quantitative description of the wealth of spectral structure observed in experiments with strongly magnetised Rydberg atoms both below and above the field-free ionisation threshold has for many years presented a lasting challenge to theory. Classical and semi-classical calculations were performed (e.g. [1-3]) to account for the appearance and spacings of quasiLandau resonances observed in the spectrum [4], and quantal calculations [5-10] were able to explain at least some general features of the spectra. It is, however, evident that the ultimate test for theory lies in the quantitative comparison between the theoretical and experimental values for the positions and intensities of the hundreds of Rydberg lines observed in a spectrum. It is the purpose of this note to carry out such a comparison in an extended, nontrivial interval of energy ranging from the beginning of the $n$-mixing

* Research supported by Deutsche Forschungsgemeinschaft (DFG) regime way up to shortly below the field-free ionisation threshold. Thus it will be demonstrated that in this essential part of the spectrum, which e.g. contains the "build-up" (Delande and Gay [11]) of the quasiLandau resonances, this "principal remaining problem in the elementary quantum mechanics of a oneelectron atom" (Kleppner et al. [12]), can be considered solved. We call this range of energy the transition region from regularity to irregularity since it is in this range that, as a consequence of the nonintegrability of the underlying Hamiltonian, in the corresponding classical system the fraction of regular orbits in phase space is steadily decreased, and from a certain energy on the classical system behaves completely chaotically. Therefore our investigations also allow addressing the more general question of how the spectrum of a quantum system behaves in a range of energy where its classical counterpart undergoes a transition to chaos. This question is of importance in the context of current efforts to define the notion of "quantum" stochasticity (see e.g. [13, 14]). 

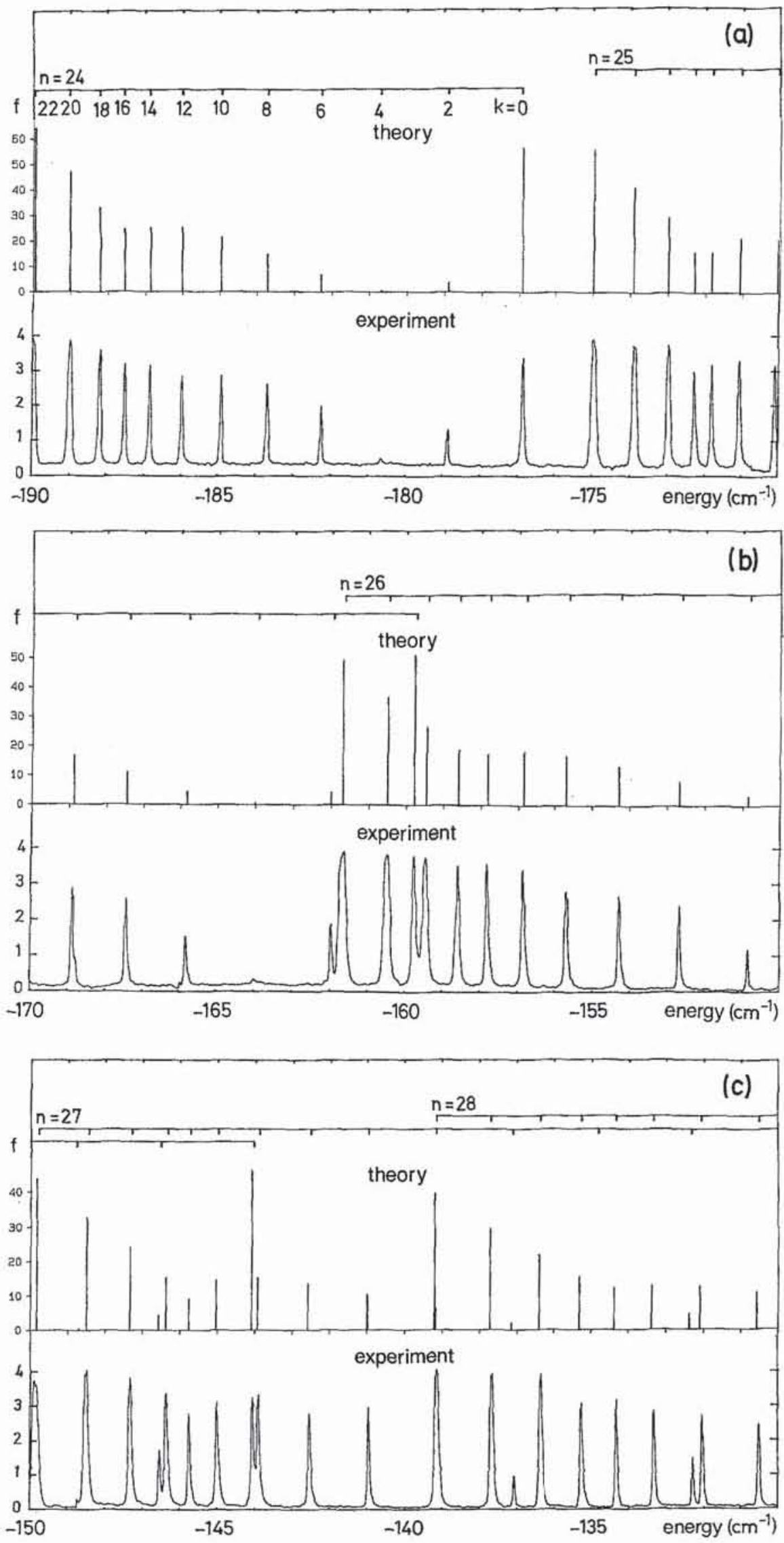

Fig. 1a-f. Deuterium Rydberg atoms in a magnetic field of $6.0 \mathrm{~T}$ : comparison between the theoretical oscillator strength spectrum and the experimental photoabsorption spectrum for $\Delta m=0$ Balmer transitions to $m=0$, even-parity Rydberg states, over the range of energy $-190 \mathrm{~cm}^{-1}$ to $-70 \mathrm{~cm}^{-1}$. Oscillator strengths are given in units of $10^{-6}$, the experimental intensity scale is in arbitrary units. The six successive energy intervals cover the range from the onset of $n$-mixing (in frame (a) the $n=24$ and $n=25$ manifolds are still just separated from each other, while in frame (b) the uppermost state of the $n=25$ mainfold lies already inside the $n=26$ mainfold) to the regime where the approximate integrability of the problem is destroyed to an ever increasing extent (frame $(f)$ ). A total of 177 lines contribute to the spectrum shown. The indices $n$ and $k$ labelling the states are the principal quantum number and the separation index to which the corresponding states can diabatically be traced back in the limit $B \rightarrow 0$ 

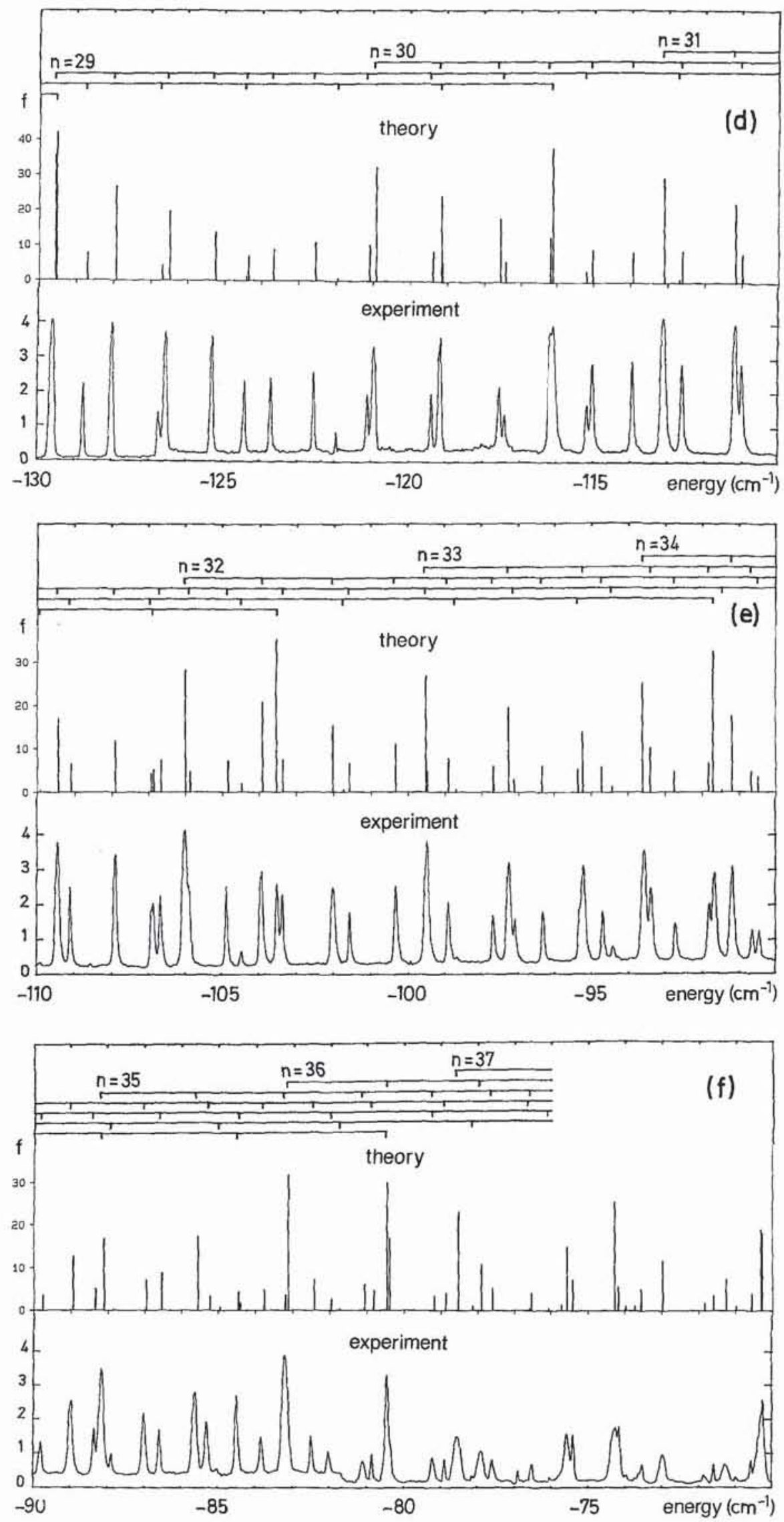
A quantitative comparison of experimental and theoretical spectra has become possible since, on the one hand, high resolution experiments with highly excited one-electron systems (hydrogen and deuterium) have been performed in $4-6 \mathrm{~T}$ fields [15], and, on the other, numerical procedures have been developed which allow an almost routine calculation of very accurate energy values, wave functions, and, thus, transition probabilities, of hydrogenic systems in uniform magnetic fields below the field-free ionisation limit. A preliminary comparison of experimental and theoretical energy values, and, partly, some oscillator strengths, was recently performed in two small sections of the energy scale [16]. We note that Delande and Gay [17] also compared sample results for a few energy values of magnetised Rydberg states with their own experimental caesium data. The objective of the present work is far more ambitious, namely the quantitative comparison for $\sim 420$ Rydberg lines in the photoabsorption spectra of deuterium atoms in magnetic fields $\sim 6 \mathrm{~T}$ from the point of view of energy and intensity.

\section{Experiment}

The experimental method used to measure the spectra is the same as in Ref. 16, and has been described recently [15]. Here we give a brief summary of the substantial experimental features. Rydberg states of even parity were prepared by resonant two-photon absorption through Paschen-Back resolved substates of $2 p$. The VUV laser light for the first excitation step $(\lambda \cong$ $121.6 \mathrm{~nm}$ ) was polarised parallel to the magnetic field, tuned to excite the $2 p, m=0$ Paschen-Back state, and kept at fixed wavelength during the measurement. The tunable UV laser light for the second excitation step $(\lambda \cong 364-366 \mathrm{~nm})$ with a band width of $\sim 0.07 \mathrm{~cm}^{-1}$ was also polarised parallel to the magnetic field. In this way pure $m=0$ final states with even parity were excited. Both laser beams were directed perpendicular to each other, and perpendicular to the well collimated beam of atomic deuterium, which was directed parallel to the magnetic field. This arrangement minimizes motional electric fields, reduces the effective Doppler width to well below the band width of the lasers, and defines an effective excitation zone of $\sim 0.5 \mathrm{~mm}$ diameter. The zone was located between fine-mesh grid electrodes, which allowed reducing electric stray fields orientated parallel to the magnetic field to below $100 \mathrm{mV} / \mathrm{cm}$. After excitation the atoms drifted, at thermal velocities, into a constant parallel electric field $(\sim 3.5 \mathrm{kV} / \mathrm{cm})$, which field-ionised them and accelerated the electrons to a surface barrier diode serving as the detector.

\section{Theory}

A different numerical approach was taken to obtain highly accurate solutions of the - nonintegrable Schrödinger equation describing an electron under the simultaneous action of a fixed Coulomb potential and a strong homogeneous magnetic field. For given magnetic quantum number $m$ we expanded the wave functions in terms of spherical harmonics, $\psi_{m}=$ $\Sigma h_{\ell}(r) \cdot Y_{\ell, m}(\theta, \phi)$, and the radial functions in a complete, orthonormal basis which is constructed from generalized Laguerre functions with fixed exponent. We have used this approach previously to explore the properties of "circular" Rydberg states in strong magnetic fields [18]. Matrix elements with respect to this basis can be expressed in closed analytical form and give rise to a banded Hamiltonian matrix which can be diagonalized by efficient standard algorithms. Our choice of basis is similar to the Sturmian basis employed by Clark and Taylor [5-7] but avoids the complications associated with the nonorthogonality of the latter. As compared to the oscillator basis in semiparabolic coordinates (used e.g. by Delande and Gay [19], and Wintgen and Friedrich [8-10], where the diagonalization produces eigenstates each of which belongs to a different value of the magnetic field strength (solutions are obtained in the $E-B$ plane along straight lines $E / B=$ const.), our basis has the advantage that one diagonalization procedure yields the spectrum at fixed $B$, in accordance with the experimental situation. In our calculations we worked with basis sizes of up to 6400 for determining both eigenvalues and eigenvectors of the first $\sim 600$ states in given $m$-parity subspaces in the strong-field regime. Convergence was established by varying the size of the basis and the exponent of the Laguerre functions. Accuracies of six significant figures can be guaranteed for energy values, and of two to three significant figures for oscillator strengths. We note that in the results to be presented below the mass scaling laws valid in a magnetic field $[20,21]$, which relate the field strength, energies, and oscillator strengths pertaining to the infinite-nuclear-mass hamiltonian to those belonging to finite core mass, are taken into account.

\section{Discussion}

Figure 1 shows a comparison between theoretical and experimental spectra for $\Delta m=0$ Balmer transitions to Rydberg states with $m=0$, even parity, and energies between $-190 \mathrm{~cm}^{-1}$ and $-70 \mathrm{~cm}^{-1}$. The magnetic field strength in the experiment was $6.0 \mathrm{~T}$. The theoretical results were obtained for a value of the magnet- 

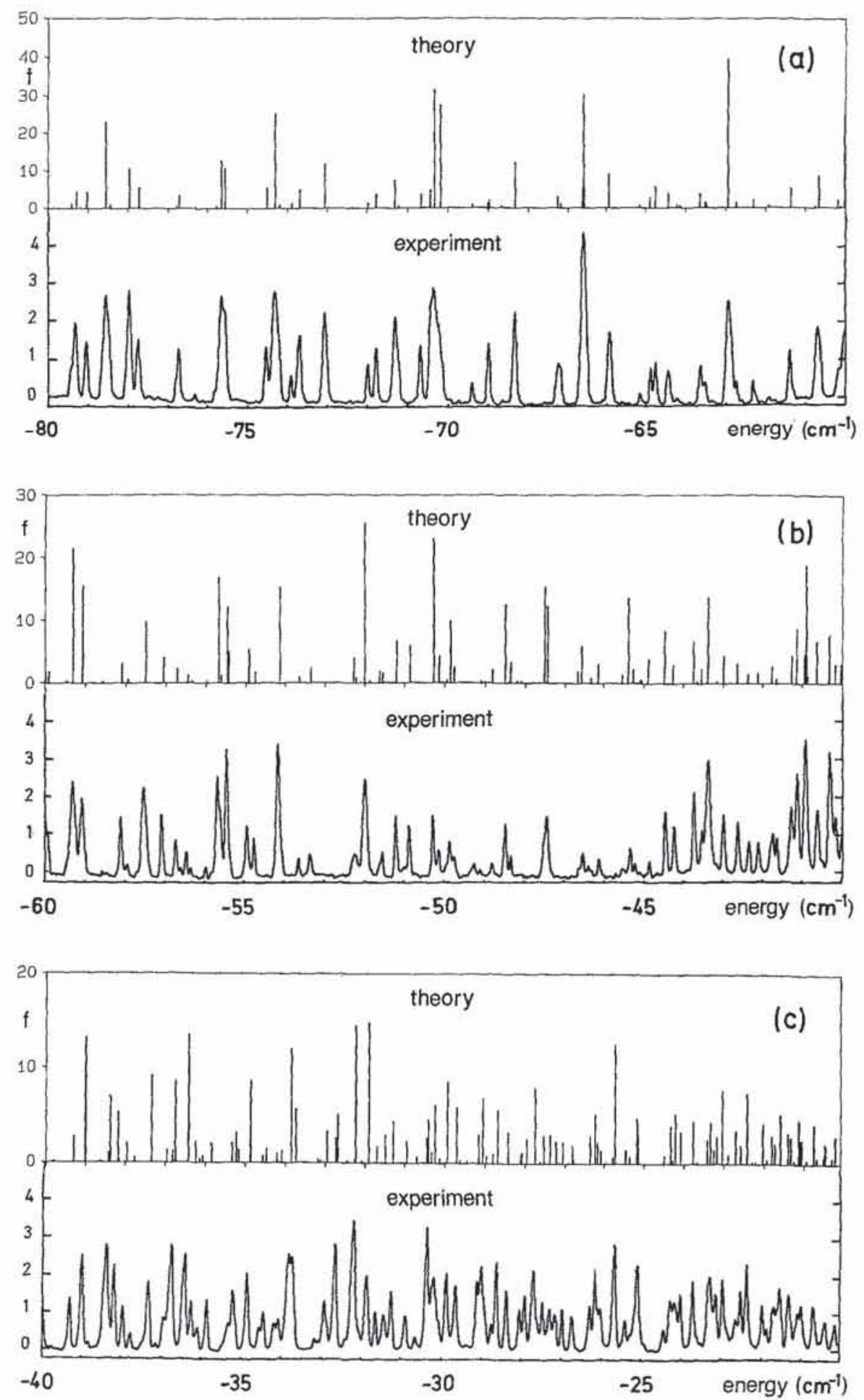

Fig. 2a-c. Same as Fig. 1 but for a magnetic field of $5.96 \mathrm{~T}$ and energies of the Rydberg states between $-80 \mathrm{~cm}^{-1}$ and $-20 \mathrm{~cm}^{-1}$ Note that at the end of the energy range (above $\sim-25 \mathrm{~cm}^{-1}$ ) the corresponding classical system becomes completely chaotic. 276 lines are shown in this spectrum

ic field parameter $\beta\left(=B / B_{0}\right.$ with $B_{0}=2\left(m_{e} c\right)^{2}$; $\left.(e h) \cong 4.701 \times 10^{5} \mathrm{~T}\right)$ of $1.275 \times 10^{-5}$ corresponding, for deuterium, to $B=5.997 \mathrm{~T}$. A total of 177 lines are compared in Fig. 1. We encourage the reader to go through the figure line by line. The agreement between the theoretical predictions and the experimental values for the positions and intensities of the lines is evidently excellent. Moreover, theory can in fact inform on where neighbouring lines were not resolved. Slight differences in the relative intensities of a few lines are due to the fact that saturation effects occurred in the experiment in strong lines, and, also, to the uncertainty on the strength of the magnetic field. 
The range of energy considered in Fig. 1 extends from the onset of the overlap of neighbouring $n$ manifolds deeply into the $n$-mixing regime. The overall structure of the spectrum still seems relatively ordered, with the clusters of lines belonging to the different $n$ manifolds interpenetrating, at least at the beginning, without substantial mutual perturbation. This behaviour, which was already pointed out in the earlier calculations of Clark and Taylor $[6,7]$, and even allows labelling the states in terms of weak-field quantum numbers, is closely related to the existence of an approximate constant of motion, and thus the approximate integrability of the problem (cf. [9]), in this range of energy.

The comparison between experimental and theoretical spectra shown in Fig. 2 proceeds to regions where this approximate integrability is rapidly destroyed, as witnessed by the disappearance of very narrow avoided crossings of the energy levels when viewed as functions of the field (cf. Fig. 3 in Ref. 16). Again we consider $\Delta m=0$ Balmer transitions to deuterium Rydberg states with $m=0$ and even parity, and the energies lie in the interval from $-80 \mathrm{~cm}^{-1}$ to $-20 \mathrm{~cm}^{-1}$. In this case the experimental data were taken at a magnetic field of $5.96 \mathrm{~T}$, and the theoretical results were determined for $\beta=1.2675 \times 10^{-5}$ $(B=5.9619 \mathrm{~T})$. A total of 276 lines contributes to the theoretical spectrum. As one moves up in energy in Fig. 2, the line density is clearly growing, and the structure of the spectrum becomes increasingly complicated. Nevertheless it is also here that we find practically complete agreement between theory and experiment, with the experiment being limited, as regards the number of detectable lines, by the finite resolution.

Figure 2 can serve as a starting point for further investigations. A Fourier analysis of the spectrum, for example, reveals that in spite of the seeming irregularity of the spectrum the line sequence does indeed contain periodic features. From the Fourier transform of the theoretical and experimental spectrum one can recognise (Fig. 3a and b, respectively) that at least periodicities of $\sim 1.7 \hbar \omega_{c}$ and $0.66 \hbar \omega_{c}\left(\omega_{c}\right.$ is the electron cyclotron frequency) are present in the spectrum. The peak marked by $\downarrow$ corresponds to the well known quasi-Landau resonances which, at $E=0$, show a spacing of $1.5 \hbar \omega_{c}$ [4]. The peak marked by $*$ corresponds to a new type of quasi-Landau resonances discovered recently [15]. These resonances show a spacing of $0.64 \hbar \omega_{c}$ at $E=0$. The differences with respect to the above values are due to the energy dependence of the resonance spacings. It should be pointed out that in the Fourier transforms of the experimental spectra which extend beyond the ionisation limit even more resonances, with spacings smaller than

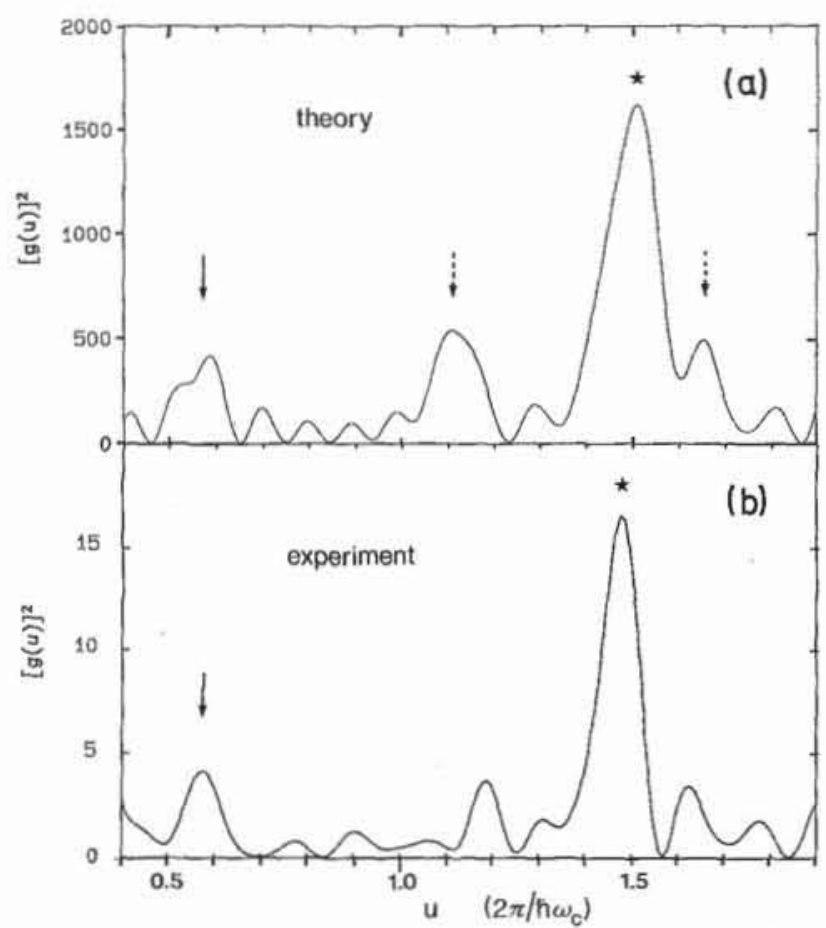

Fig. 3a and b. Square of the Fourier transform of the theoretical (a) and experimental (b) spectrum shown in Fig. 2. The unit of the abscissa is chosen in such a way that a periodicity of $\gamma \cdot h \omega_{e}$ in the original spectrum is reflected by a peak at $1 / \gamma$ in the Fourier spectrum. Thus the feature around $u \sim 0.57$, together with its two harmonics (dashed arrows), corresponds to $\gamma \sim 1.7$, while the more prominent feature at $u \sim 1.5$ corresponds to $\gamma \sim 0.66$

$0.64 h \omega_{c}$, have been found, and have theoretically been accounted for by classical trajectory calculations [22]. So far no exact quantum mechanical results are available at $6 \mathrm{~T}$ above the ionisation limit. The existence of the Fourier peaks in Fig. $3(\downarrow$ and $*$ ) clearly indicates the build-up of the corresponding quasiLandau resonances below the ionisation limit.

To investigate a possible connection to chaotic behaviour in the corresponding classical system we computed classical trajectories for $B=6 \mathrm{~T}$ and $m=0$ as a function of energy. We find [23] that the first irregular orbits appear around $\sim-100 \mathrm{~cm}^{-1}$, and the last regions filled with regular orbits vanish in the Poincaré surface of section at $z=0$ around $-25 \mathrm{~cm}^{-1}$, which therefore is the critical classical energy of the onset of chaos for the set of parameters chosen. Thus in Fig. 2 one actually has the opportunity of watching the spectrum of a quantum system evolve in a range of energy where its classical counterpart undergoes a transition to irregularity. However, a mere inspection of the spectrum does not exhibit any perspicuous differences between the structure of the spectrum below and above the critical energy. To find such differences it is necessary to look at more basic properties of the spectrum, e.g. the statis- 
tics of the level sequences in different parts of the spectrum, which display a transition from a Poissonlike to a Wigner-like distribution as one proceeds to ranges of energy where classical motion becomes increasingly irregular [23-25]. This underlines the fact that magnetised Rydberg atoms are indeed promising objects in the current search for manifestations of stochasticity in quantal systems.

To summarize, in this paper a complete experimental spectrum in the strong-field regime has successfully been accounted for, in quantitative terms, by the positions and oscillator strengths of the corresponding quantum theoretically calculated (in total 426 different) photoabsorption lines. This certainly can be considered essential progress in the quantitative spectroscopy of highly excited atoms in strong magnetic fields.

\section{References}

1. Starace, A.F.: J. Phys. B: At. Mol. Phys. 6, 585 (1973)

2. Rau, A.R.P.: J. Phys. B: At. Mol. Phys. 12, L 193 (1979)

3. Reinhardt, W.P.: J. Phys. B: At. Mol. Phys. 16, L 635 (1983)

4. Garton, W.R.S., Tomkins F.S.: Astrophys. J. 158, 839 (1969)

5. Clark, C.W., Taylor, K.T.: J. Phys. B: At. Mol. Phys, 13, L737 (1980)

6. Clark, C.W., Taylor, K.T.: J. Phys. B: At. Mol. Phys. 15, 1175 (1982)

7. Clark, C.W., Taylor, K.T.: J. Physique C2 supply. 11 43, 19 (1982)

8. Wintgen, D., Friedrich, H.: J. Phys. B: At. Mol. Phys. 19, 991 (1986)

9. Wintgen, D., Friedrich, H.: J. Phys. B: At. Mol. Phys. 19, 1261 (1986)

10. Wintgen, D., Friedrich, H.: J. Phys. B: At. Mol. Phys. 19, L99 (1986)

11. Delande, D., Gay, J.C.: Phys. Lett. 82 A, 399 (1981)

12. Kleppner, D., Littman, M.G., Zimmerman, M.L.: Rydberg atoms in strong fields. In: Rydberg states of atoms and molecules. Stebbings, R.F., Dunning, F.B. (eds.), pp 73-116, Cambridge: Cambridge University Press (1983)

13. Bohygas, O., Giannono, M.J., Schmit, C.: Phys. Rev. Lett. 52, 1 (1984)

14. Casati, G. (ed.): Chaotic Behaviour in Quantum Systems. New York: Plenum Press 1985

15. Holle, A., Wiebusch, G., Main, J., Hager, B., Rottke, H., Welge, K.H.: Phys. Rev. Lett. 56, 2594 (1986)

16. Wintgen, D., Holle, A., Wiebusch, G., Main, J., Friedrich, H., Welge, K.H.: J. Phys. B: At. Mol. Phys. 19, L557 (1986)

17. Delande, D., Gay, J.C.: J. Phys. B: At. Mol. Phys. 19, L173 (1986)

18. Wunner, G., Kost, M., Ruder, H.: Phys. Rev. A33, 1444 (1986)

19. Delande, D., Gay, J.C.: J. Phys. B: At. Mol. Phys, 16, L 335 (1984)

20. Pavlov-Verevkin, V.D., Zhilinskii, B.I.: Phys. Lett. 78A, 244 (1980)

21. Wunner, G., Ruder, H., Herold, H.: Astrophys. J. 247, 374 (1981)

22. Main, J., Wiebusch, G., Holle, A., Welge, K.H.: Phys. Rev, Lett. $\mathbf{5 7 ,} 2789(1986)$

23. Wunner, G., Woelk, U., Zech, I., Zeller, G., Ertl, T., Geyer, F., Schweizer, W., Ruder, H.: Phys. Rev. Lett. 57, 3261 (1986)

24. Wintgen, D., Friedrich, H.: Phys. Rev. Lett. 57, 571 (1986)

25. Delande, D., Gay, J.C.: Phys. Rev. Lett. 57, 2006 (1986)

A. Holle

G. Wiebusch

J. Main

K.H. Welge

Fakultät für Physik

Universität Bielefeld

D-4800 Bielefeld

Federal Republic of Germany

G. Zeller

G. Wunner

T. Ertl

H. Ruder

Lehrstuhl für Theoretische Astrophysik

Universität Tübingen

D-7400 Tübingen

Federal Republic of Germany 